\title{
Apprentissages informels dans l'activité : dispositif de participation et processus d'engagement du remplaçant en masso-kinésithérapie
}

Learning on the job: involvement structure and engagement process. The case of people substituting for physiotherapists

Aprender en el trabajo: dispositivo de participación y proceso de compromiso. El caso de los sustitutos en masajes de kinesioterapia

Bei der Arbeit lernen: Teilnahme und Prozess des Engagements. Der Fall der Vertreter in Krankengymnastik

\section{Paul Olry}

\section{(2) OpenEdition}

\section{Journals}

Édition électronique

URL : http://journals.openedition.org/rfp/592

DOI : 10.4000/rfp.592

ISSN : 2105-2913

\section{Éditeur}

ENS Éditions

\section{Édition imprimée}

Date de publication : 1 septembre 2007

Pagination : $39-50$

ISBN : 978-2-7342-1096-2

ISSN : 0556-7807

\section{Référence électronique}

Paul Olry, «Apprentissages informels dans l'activité : dispositif de participation et processus

d'engagement du remplaçant en masso-kinésithérapie », Revue française de pédagogie [En ligne], 160 | juillet-septembre 2007, mis en ligne le 01 septembre 2011, consulté le 23 mars 2021. URL : http:// journals.openedition.org/rfp/592 ; DOI : https://doi.org/10.4000/rfp.592 


\section{Apprentissages informels dans l'activité : dispositif de participation et processus d'engagement du remplaçant en masso-kinésithérapie}

Paul Olry

Chacun convient que l'on peut apprendre en travaillant, bien que la nature de cet apprentissage soit parfois difficile à définir. C'est dire que des apprentissages informels se réalisent dans le cours de l'activité professionnelle. L'étude présentée ici s'appuie sur l'analyse des échanges lors des remplacements d'un praticien kinésithérapeute avec son remplaçant. Les dimensions non formelles de l'activité émergent lors de leur mise au débat. La première condition est l'existence d'un dispositif de participation à l'activité, la seconde un engagement conjoint du remplaçant. Notre proposition est ici que le support de leur formalisation se trouve dans le passage entre modèle opératif et modèle cognitif de l'activité de soin. L'article passe enfin en revue quelque processus supports de cette formalisation.

Descripteurs (TEE) : apprentissage fortuit, construction de modèle, identité professionnelle, remplacement temporaire, savoir-faire.

'objet de cette contribution est de s'attacher au(x) processus de mises au jour et de formalisation d'apprentissages informels au travail qui se révèlent le plus souvent par la performance atteinte, mais dont on ne sait pas d'emblée restituer l'élaboration. Autant les modalités d'apprentissage de savoirs et de savoir-faire formalisés sont en effet bien étudiées, notamment au travers des didactiques, autant d'autres apprentissages restent largement informels. Cette appellation couvre une diversité de savoirs, de connaissances et de dispositions acquises qui, dans le jeu social autour du travail et de la formation, ne font pas l'objet d'une intention explicite de transmission. Peu d'auteurs s'y sont attachés, mais on en trouve une étude, du point de vue du travail, dans le Work Process Knowledge (Boreham, Fischer \& Samurçay, 2002), tandis que O. Charbonnier et P. Carré (2004) en précisent les conditions du point de vue du sujet en terme de motivation et d'engagement du sujet.

Or les connaissances relatives aux exigences du travail et des pratiques, sont souvent au principe même de l'efficacité de l'action. En effet, elles ne relèvent pas seulement d'une acquisition individuelle mais de médiations assurées tant par les objets que 
par les pairs. Informel, cet apprentissage incident se prête mal à une pédagogie ou à un choix de contenus qui serait opéré par ceux dont le métier est de formaliser des savoirs-faire à des fins de transmission.

Le paradoxe est que les formalisations propres aux métiers du soin, tels que les protocoles, les procédures ou les modes opératoires, participent de ces apprentissages informels, dont l'origine et les voies d'acquisition sont réputées découler de l'expérience. Ces descriptions, prescriptions ou indicateurs de performance jouent une fonction de clôture sociale des pratiques de métier, en établissant les frontières de ce qui se fait (et ce qui ne se fait pas), sans garder la trace de la façon dont ce savoir, cette conceptualisation se sont constitués.

Nous nous attachons à montrer d'abord l'enjeu que recouvre l'investigation de ce type d'apprentissages pour le développement du métier. Nous envisageons ensuite les conditions sociales de leur émergence, en prenant appui sur l'analyse du couple «participation »/« engagement » (Billet, 2002 \& Thévenot, 2006) des remplaçants à l'action thérapeutique sur la base d'une étude réalisée avec des professionnels de masso-kinésithérapie.

Enfin, prenant appui sur la distinction modèle opératif - modèle cognitif de l'action (Pastré \& Weill-Fassina, 2004) -, nous proposons une clarification de quelques processus contribuant à la formalisation d'apprentissages informels, qui constituent une voie de développement des compétences professionnelles.

\section{APPRENTISSAGES INFORMEL (1) ET NON-FORMEL, INGRÉDIENTS DE L'EXERCICE DU MÉTIER}

Parmi les allants de soi sur l'apprentissage d'un métier, chacun est convaincu que tout ne peut être formalisé. II est convenu qu'avec l'expérience, les fondamentaux transmis dans un cadre intentionnel de transmission fructifieront pour " faire " un bon professionnel. II en est ainsi de la formation des masseurskinésithérapeutes, qui comporte des temps de stages en hôpitaux ou en centres de rééducation fonctionnelle. Autant dire que l'action du stagiaire s'inscrit alors dans un cadre socio-organisationnel complexe. Or, à $80 \%$, ces stagiaires diplômés d'État s'engagent dans un exercice libéral, dont le contexte est radicalement différent des modalités d'exercice qu'ils ont connu en stage (2). C'est pourquoi dans leur grande majorité, les jeunes professionnels intègrent un cabi- net en tant que remplaçant ou associé. Dès lors, ils entrent dans une phase d'intégration non seulement dans le cabinet mais aussi dans le métier.

Le moment emblématique de cette intégration est la prise en charge personnelle d'un patient dont on assume la responsabilité de la rééducation pour la première fois.

\section{L'informel lié à la relation au patient}

À l'occasion des séances, les remplaçants (3) découvrent à quel point le soin ne relève pas seulement d'un système d'observations cliniques, de prévisions thérapeutiques clairement intégrées et hiérarchiquement structurées, mais du partage d'un système de croyances, mêlant les schémas du patient et parfois les connaissances du praticien pour la réussite du soin.

Les gestes thérapeutiques pratiqués jouent alors une fonction "d'intercalaire social » sans autre finalité que la poursuite d'une compréhension partagée du soin et de ses exigences. L'efficace (4) du soin ne reposerait pas seulement sur l'efficacité du geste. Les professionnels sont là pour comprendre les expériences quotidiennes du patient et traiter ces informations factuelles dans le cours de leur action, en contrepoint de savoirs scientifiques aux contenus hautement formalisés. S'il apparaît difficile d'opérer cette liaison, c'est qu'au plan clinique, le professionnel se trouve parfois en difficulté d'expliquer, non son geste, mais l'efficacité qui lui est attachée, à tel moment précis, et donc les préconisations qui lui sont associées.

La situation de soin, ensuite, inscrit l'activité du praticien dans une relation au sein de laquelle le patient ne joue pas toujours le même rôle :

- le patient est d'abord objet du travail. Sa présence implique l'engagement subjectif du praticien dans son activité ;

- le patient est ensuite sujet du travail : l'objet est un sujet. Si l'on peut donc affirmer que la pratique de l'art du soin kinésithérapique prend appui de façon discontinue, mais non aléatoire, sur le patient, pour autant celui-ci n'est pas toujours en position d'objet du soin.

Ainsi, le patient est appréhendé de différentes façons par le kinésithérapeute :

- c'est d'abord un corps qui «parle", puisque les doigts du thérapeute "l'écoutent" (Olry, 2003, p. 41). Ce contact quotidien au patient, d'un corps à l'autre, est une affaire incertaine, que la maîtrise 
d'un ensemble de savoirs disciplinaires (anatomie, biomécanique, physiologie) vise à clarifier en affirmant des connaissances assurées et des relations de causalité certaines (5) ;

- c'est ensuite une personne qui s'exprime, contraignant le soignant à contenir ou à orienter le flux verbal du patient vers ce dont il peut faire usage (Olry et al., 2005) ;

- c'est enfin un objet qui (se) trahit dans la mesure où se taire, craquer, crisper, à son corps défendant, sont autant de manifestations des difficultés d'ajustement entre les protagonistes de cette interaction.

Quand on agit sur des objets qui sont des sujets, on peut agir sur, avec, contre, ou sans l'objet (Gadrey \& De Bandt, 1994 ; Hughes, 1958). II y a du travail, des échanges, des problèmes, des sollicitations, des engagements de soi réciproques qui dépassent une action seulement dirigée vers le patient. Dit autrement, l'application d'une thérapeutique, avant de concerner l'éducation du patient est une école pour le soignant, dont les processus restent à clarifier.

\section{L'informel lié aux situations cliniques}

Au premier abord, la relation de soin, patient/kinésithérapeute semble largement structurée par les rôles respectifs à tenir : un soignant qui sait ce qu'il fait et un patient docile aux manipulations et mobilisations engagées. À la catégorisation spécifiant les degrés de déficit du patient, répondent des degrés de maitrise, des rôles et niveaux de savoir-faire des soignants dans l'action. Mais, dans leur pureté, ces classifications prennent peu en compte la singularité de situations, dans lesquelles soignant et patient, conjointement engagés sur des buts différents, s'ajustent dans la perspective de produire les effets attendus du soin.

Une spécificité singularise la pratique kinésithérapique : le corps du patient est un point d'appel pour un traitement. Le soin se construit donc dans une relation personnelle, dans l'interaction avec le patient en tant que sujet, et dans une confrontation continue avec le déficit fonctionnel d'un corps aux réactions infiniment variables. Nombre de savoirs d'expérience sont construits chemin faisant par le praticien et ne font pas l'objet de descriptions (6).

Cette variété de cas et cette absence de descriptions posent au praticien un problème de conscience de la situation, par exemple entre la nécessité d'appliquer la thérapeutique liée à la prescription et la nécessité de soulager la gêne des patients.
En effet le patient est aussi un client, en attente d'un service, autant éducatif que thérapeutique, tout au long du déroulement des séances.

Au contact des patients, la pratique quotidienne d'une large majorité des praticiens libéraux, prend appui sur les indications qui fondent les recommandations professionnelles (7). Mais les mobiliser suppose de les configurer, en sélectionnant et en ordonnant les savoirs pertinents (i. e. actualisés dans la rencontre de corps et de sujets "résistants "). Ainsi, en soignant un patient, de nouveaux buts émergent qui modifient l'activité, son adresse et son destinataire. Les remplaçants sont ainsi confrontés à l'informel d'une clinique des relations intersubjectives.

\section{L'au-delà et l'en deçà des formalisations disponibles}

A priori, la question de la formalisation ne se pose pas en kinésithérapie. De multiples ouvrages existent qui décrivent, souvent sous forme de cas cliniques, des problèmes traitables par des protocoles constitués. Mais chaque situation est particulière et questionne les répertoires d'action, les pattern familiers car la réalité des situations rencontrées leur est difficilement superposable : comment je fais, non pas en général, mais avec ce patient ?

Pour ce faire, la pratique kinésithérapique mobilise un couple vertueux entre conformisme procédural et aménagements personnels des techniques. Plus ces aménagements sont fréquents, plus les procédures sont ressenties comme nécessaires. Inversement, l'alignement sur la procédure heurte parfois les corps qui résistent, parce que cette mise en œuvre de pratiques passe-partout correspond à la tentative de "se tenir » à un protocole, à défaut de savoir ou de pouvoir s'y ajuster.

Cela conduit les syndicats professionnels à proposer une prescription renforcée du travail en décrivant des "scripts comportementaux", et en préconisant de la procédure pour faire face à toutes les éventualités (8). Mais la faible détermination de la prescription médicale, le relatif isolement des masseurs-kinésithérapeutes libéraux conduisent à des aménagements des protocoles, des pratiques qui sont autant de transgressions. Chaque masseur-kinésithérapeute "se débrouille" en effet dans la multiplicité des procédures et protocoles pour adapter une pratique standardisée à chaque cas individuel, tout en restant soi-même, le cas échéant en évitant des soins que l'on n'est pas sûr de complètement maîtriser. Ainsi, 
face à la difficulté persistante d'expliciter ce qui fait l'efficace du métier, la tendance est de créer toujours plus de procédures.

Cette perspective nous indique qu'agencer les éléments déterminants de la qualité du soin à prodiguer, tels la relation au patient, les situations cliniques, les protocoles de soin comme artefacts, est une activité à construire qui comporte une large part informelle.

Dès lors, on peut interroger les processus qui contribuent à leur formalisation et appropriation.

\section{LE REMPLACEMENT : RENCONTRE D'ACTIVITÉS, OPPORTUNITÉ D'APPRENTISSAGES}

Parler de processus de formalisation, c'est faire le pari que l'informel n'est pas toujours destiné à le rester. Parmi les modalités d'appropriation de ce savoirfaire d'agencement, le remplacement en est une dont on oublie souvent que c'est l'occasion de croiser les points de vues tant pour le praticien aguerri par l'expérience que pour le remplaçant pourvu du savoir le plus actuel.

«Remplacer» n'est pas seulement exécuter une tâche attendue par les patients. C'est aussi un temps rituel d'introduction dans l'espace des professionnels aguerris, dans lequel le remplaçant se doit de tenir sa place, en adoptant et défendant un point de vue. C'est encore l'occasion, rare dans le contexte d'une activité professionnelle le plus souvent solitaire, d'un changement de position, le remplaçant agissant en lieu et place du praticien. C'est donc le moment d'une rencontre d'activités, de « façons de faire » qui s'éprouvent.

Si dans le remplacement, il s'agit de faire en lieu et place d'autrui, ce n'est pas faire comme autrui. Le faire du remplaçant est dans ce dispositif étroitement dépendant de la participation (Billet, 2002) autorisée au remplaçant, participation ici entendue tant au plan opératoire de contribution au soin qu'au plan identitaire de participation au métier à travers la «place» requise (ou prise) dans la conduite du soin.

Dans ce cadre l'action du remplaçant participe d'un but commun, "rétablir les fonctions du patient", poursuivi tant dans l'acte thérapeutique que dans la mise en débat des choix thérapeutiques de chacun. Participer c'est donc aussi prendre part à une activité collective, dont chaque sujet n'est qu'un des rouages (9).
Notre hypothèse est que ce qui produit un apprentissage informel est pour le duo praticien/remplaçant le passage entre les modèles cognitif et opératif de l'action, entre la mobilisation du geste efficace et la convocation de ce qui l'explique dans les interactions praticiens remplaçants. Ces modèles sont ouverts l'un sur l'autre, en ce sens « qu'un modèle cognitif abstrait réfère néanmoins à des situations, de même qu'un modèle opératif très pragmatique comporte des éléments conceptuels sous forme d'objets, de propriétés de ces objets et de relations entre objets et propriétés 》 (Pastré \& Weill-Fassina, 2004 ; souligné par les auteurs). Les relations entre ces deux modèles peuvent être très variées, depuis l'«ignorance mutuelle » jusqu'à la convergence par double étayage, en passant par la contradiction et le conflit. La question pour un apprentissage tient à la manière dont ces deux modèles peuvent se coordonner.

Par l'actualisation du soin qu'elle provoque, par les dilemmes, les possibilités d'échanges, voire de controverses et de débats qu'elle suscite, la participation du remplaçant conduit ainsi chacun à formuler des arguments, des raisonnements, témoins de modèles antérieurement constitués qui trouvent ici l'occasion d'une publicité.

La notion d'engagement apparaît comme une seconde condition à la possibilité de formalisation. Cet engagement est bien sûr dans l'action. Mais il est évidemment au cœur du dispositif social du remplacement, " contraignant » chacun des participants à recourir à un registre de justification qui appelle des principes, des mondes faits d'objets et des dispositifs conventionnels.

C'est pourquoi notre démarche générale s'inspire de l'approche pragmatique en sociologie (Boltanski \& Thévenot, 1991). Notre hypothèse est que la stabilité du soin prodigué, malgré le remplacement et les tensions qu'il induit sur le soin, n'est tenable que dans un régime d'engagement (Thévenot, 1999 \& 2006) qui dépasse les logiques d'action de chacun. Un régime suppose des compétences particulières des acteurs et figure comme une modalité spécifique de coordination et de traitement du monde, ayant pour finalité la clôture d'un espace de référence, au sein duquel le sujet est engagé, ce qui permet de spécifier le régime adopté.

Par delà leurs différences d'appréciation, praticien et remplaçant peuvent se distinguer par ce qu'ils entendent par «soigner pertinemment le patient», bien commun partagé tant du point de vue opératoire, que du point de vue identitaire. Ainsi, plus que le but, 
la représentation du bien gouverne « l'appréciation de ce qu'il advient et la prise en compte des retours de la réalité » (Thévenot, 1999, p. 229).

C'est donc au travers de l'activité et des tensions qui la traversent que peut être étudié le rapport à l'épreuve de réalité qui permet d'identifier chaque régime (selon le réalisme de l'épreuve et le mode d'épreuve). Les données présentées ici reposent sur une étude exploratoire, dont le matériel a été recueilli entre 2004 et 2006, et portant sur l'analyse de l'activité de masseurs kinésithérapeutes libéraux (10). Cette analyse des théorisations des praticiens se fonde autant sur les ressemblances et différences de pratiques de soin, que sur l'observation des façons dont elles se singularisent en " prenant corps » chez les remplaçants. Le matériel discuté relève avant tout de la confrontation de points de vue sur les options thérapeutiques prises au regard de cas/patients, ce qui renvoie aux coordinations, aux engagements dans l'action débattue et aux mondes de référence.

On a observé d'abord le déroulement de l'action, constituée de gestes et d'usages du langage dans un but thérapeutique. Nous avons filmé l'action en situation, lors de séances de soin qui ont donné lieu à auto-confrontation sur deux axes: l'ensemble gestes/verbalisations a donné lieu à explicitation et fourni matière à controverses entre praticien et remplaçant.

On a tenté ensuite d'appréhender la construction de la situation (11) par les soignants, lorsqu'ils apprécient les éléments saillants du tableau clinique qu'ils sélectionnent pour agir, ce choix étant l'objet de débats et de dilemmes entre eux.

Notre attente était, le débat s'établissant entre professionnels, de pister les éventuelles formalisations produites dans l'interaction. Nous considérons ainsi que cette situation de remplacement entraîne une double tension: l'une, centripète, visant à une conformation des pratiques du remplaçant. L'autre, centrifuge, tendant à l'émergence de nouvelles interprétations du cas, introduites par la participation du remplaçant.

\section{LE SOIN : TEMPS DE LA PARTICIPATION, ESPACE DE L'INFORMEL}

Les manifestations d'apprentissages informels s'inscrivent dans un double espace de tensions :
- le rapport engagement/participation qui en conditionne l'émergence ;

- le rapport modèle cognitif/modèle opératif dont la coordination fixe le format d'expression ;

- qu'en est-il pour les binômes remplaçants/praticiens entendus?

\section{Le contexte de remplacement : un mode de coordination formel pour d'éventuels apprentissages informels}

Le recours à un remplaçant peut-être le résultat d'une offre et d'une demande. Parfois, c'est aussi un parcours qui se construit dans la durée. Le remplaçant est un ancien stagiaire et peut-être deviendrat-il associé. Le récit par certains praticiens de leur entrée dans le métier, parfois reproduite dans leur façon d'accueillir les remplaçants, mentionne ainsi plusieurs bornes de ce parcours. La participation est alors l'amorce d'une histoire qui débute par des périodes de stage.

Ainsi le remplacement concerne simultanément la question d'une maîtrise et celle d'une place (ou d'un pouvoir) censée lui correspondre. Ce qui implique que le remplaçant se saisisse et dépasse les actes d'attribution d'identité venant du praticien, par une revendication d'appartenance et de qualités professionnelles. C'est pourquoi, en cabinet, le processus de formalisation est activé par l'enjeu de reconnaissance professionnelle, malgré l'asymétrie de position avec le praticien.

Nous avons constaté des différences quant aux conditions de remplacement qui contraignent évidemment la posture des professionnels, lors de la mise en discussion de cas/patients.

Dans le premier contexte, la participation tolérée du soignant s'inscrit dans une attente de conformité, qui est ordonnancée par le praticien en fonction des moments, des traitements à assurer. Le terrain est en quelque sorte préparé, à l'intention des patients et du soignant. Les soins à prodiguer aux cas «les plus lourds » ont été planifiés, en tenant compte de l'absence du praticien. L'agenda des soins se trouve aménagé pour ne proposer au remplaçant que des séances de mi-parcours ou au contraire de nouveaux patients, «rattrapables » en quelque sorte par le praticien.

Dans le deuxième contexte, le praticien et/ou le remplaçant peut au contraire adopter une stratégie opposée pour certains patients. Si le traitement en 
cours «flotte», si le praticien sait (ou se doute) que son traitement n'est pas optimal, l'action du remplaçant se présente alors comme une alternative pour débloquer la situation. La participation autorisée du remplaçant modifie le système d'attentes propres au praticien et de ses patients.

Un troisième contexte est celui de la prise d'initiative du soignant qui, par delà ce qui lui a été préparé, ce qui lui est " autorisé », se pose et s'impose, quitte à bousculer un ordre de séance, le choix d'un protocole, etc.

L'action du remplaçant introduit donc de la variation dans un système stabilisé par le praticien, dont les effets dépassent le domaine des techniques de métier pour influer sur le système « praticien/patient », notamment la participation de ce dernier à la relation de soin asymétrique (Houel, 2002). Cette variation fonde une "lecture " différente voire discordante du cas clinique. Cette participation prise dans le traitement par le remplaçant s'énonce dans les séances de soin par la communication thérapeutique - échanges langagiers et les gestes de soin -, forme de dialogue croisé avec le patient et avec le praticien même hors de sa présence.

La tenue et la poursuite de ce dialogue supposent une coordination, matérialisée par un partage d'informations (12) voire des contributions réciproques, tant en terme d'actions que d'argumentations autour du traitement à choisir, puis appliquer. Cette activité de coordination est alors moins dirigée vers le patient que vers le pair.

\section{Les formes de circulation entre modèle cognitif et modèle opératif}

Ainsi que nous l'avons évoqué plus haut, chaque patient est un défi pour le remplaçant. L'inscription des situations, les événements cliniques qui surviennent, sont autant d'épreuves qui mobilisent pour être dépassées l'espace des expériences antérieures. De celui-ci les soignants retirent des modèles plus ou moins affinés, comportant de nombreuses variantes, qu'ils évoquent non de façon exhaustive mais le plus souvent sous la forme de règles d'action ou de schémas d'action (Sonntag, 2002).

L'ignorance mutuelle des modèles : la confrontation procédure (issue de la formation)/règle d'action (tirée de l'expérience pratique)

Ici, la formalisation d'apprentissages informels antérieurs ressort de la nécessité d'élucider l'action, en évoquant l'expérience directe du contact, commune aux gens de métier, à l'aide des concepts explicatifs des corps, des espaces thérapeutique, gestes, etc., bref des références minimales communes.

R1 (13) : Je place le coussin; souvent je fais comme ça quand je m'occupe de l'épaule: comme ça, ils ne sont pas trop en rotation externe; ça me permet d'avoir l'épaule comme je l'ai apprise en anatomie et trouver les bons tendons, pas tout à fait dans la même position, mais plus proche.

La discussion autour des positions dans lesquelles le patient est placé, manifeste un recours à l'anatomie - savoir explicite -, mais également un savoir d'action, l'accès aux «bons tendons [...] plus proches". Ce à quoi le praticien répond en s'inscrivant dans le discours de la formation, mais en évoquant la douleur du patient, lui redonnant ainsi une place de sujet.

P1: Mon problème à moi c'est que j'ai quelqu'un qui souffre entre 8 et 3 (EVA), 8 pour elle c'est quand même quelque chose de lourd pour elle, parce qu'on était tombé largement au dessous de 3 avec ça (l'EVA) et ressent une gêne fonctionnelle importante. Mon objectif, ma problématique c'est de supprimer la douleur, récupérer la mobilité et donc récupérer une fonction normale pour retrouver une mobilité fonctionnelle normale.

Au remplaçant qui apprécie le cas/patient en évoquant la procédure et son respect, le praticien répond par l'affirmation de son engagement auprès d'un patient avec un but précis « supprimer la douleur [...] récupérer la mobilité. " L'engagement du remplaçant semble subordonné à son expérience scolaire, laquelle le conduit à rechercher des postures connues (« comme je l'ai apprise »), ce qui apparaît cohérent avec les modalités de participation qui lui sont ouvertes par le praticien. Ces deux modèles cognitifs, légitimes s'ignorent dans ce mode de coordination.

La convergence et l'étayage des modes opératoires et des protocoles de l'action

Une seconde source de formalisation a pour origine les modèles disponibles de l'action. Mais parmi celles-ci encore faut-il choisir «l'action qui convient ». L'usage de ces formalisations se forge à l'épreuve du patient dans une sorte de logique d'exercice et constitue un savoir intermédiaire néanmoins explicitable.

Ainsi, un jeune néo-titulaire évoque-t-il à sa façon une redéfinition du mode opératoire qu'il adopte :

R3 : Alors, toujours en début de séance toujours savoir quelle sont les douleurs qu'il y a eu entre les 
deux séances... pour savoir si... il y a des progrès au niveau de la douleur et pour savoir si je vais pouvoir tirer dessus : c'est une butée de Latarget qui a trois semaines. Il en est à sa septième séance sur... il doit $y$ en avoir douze ou quinze.

Là encore, les références du praticien diffèrent signalant l'attention à porter aux indicateurs choisis :

P3 : Oui, c'est vrai, ...Au départ ça appartient à un système rangé. Mais si ça ne va pas, il y a déclenchement d'une enquête. Par exemple ici, pour moi, il faut travailler la qualité du craquement. II y a plusieurs sortes de craquements : est-ce que je l'entends une fois ou s'il y a reproduction au mouvement? est-ce que cela correspond à une surface articulaire irrégulière ou à un claquement? est-ce que j'ai une perception grain de sable sous les doigts?

Ces différences, à défaut d'être débattues, finissent par constituer un point de vue implicite sur la situation de soin, qui cadre l'action et l'attitude du praticien.

R1 : Donc je vais repérer les éléments qui gênent et ce n'est pas un conflit sous-acromial. La preuve c'est que j'ai soigné des éléments qui sont complètement de l'autre côté, qui sont au niveau de... de la.... scapule arrière et c'est une tendinite de position, avec un problème de trapèze etc. C'est pas du tout un conflit.

P1 : Oui je comprends. Mais tu sais, je crois qu'on apprend à mieux cibler, à mieux... et je crois qu'on apprend aussi par l'écoute, à mesurer que finalement on trouve qu'il y a des grandes fonctions qui sont souvent perturbées. Après on a tendance à techniquement développer sa technique parce qu'on sait que pour un grand thème [fonctionnel] comme ça, finalement, il y a une multitude de causes qui font que ces choses-là se trouvent déficientes.

Le praticien signale ici à son remplaçant d'une part que tout ne peut être rapporté à des répertoires de situations d'action et d'autre part que de nombreuses conceptions, collectivement partagées restent peu stabilisées. Mais surtout un dialogue s'établit dans ce type de coordination, d'abord sur les modèles opératifs mobilisés dans l'action, puis également sur les modèles cognitifs (ici, du craquement).

\section{La contradiction des modèles liée à la singularité des cas}

La prescription médicale de rééducation ne formule pas toujours une prescription claire et concise, tant par le médecin que par le patient. L'intervention kinésithérapique comporte une large part d'interpréta- tion pour concevoir la séance. Ainsi, deux soignants n'aborderont pas un patient par le même angle, sous la même perspective.

P2 : Ma remplaçante a traité deux fois sa sciatique ; son épaule lui faisait plus du tout mal, mais par contre sa sciatique oui. Alors en fait, on va se retrouver avec sur quatre séances, deux séances qui ont porté sur sa sciatique. Ça, c'est une problématique qu'on a de plus en plus. Les gens ont une sorte de "bon pour» une remise en forme chez le kiné, alors un coup on va faire une épaule, on va faire une hanche, etc. C'est la complexité de l'affaire.

II en va ainsi des gestes thérapeutiques dont on a vu les degrés d'incorporation par les praticiens (Olry et al., 2005). Ce qui est vrai pour des éléments du geste thérapeutique l'est également dans la manière de prendre la parole auprès du patient, en lien avec les protocoles adoptés:

R2 : J'ai l'impression que ce qui compte, peut-être ce qui ne peut pas s'apprendre au sens savoir-faire, c'est d'arriver à se rendre compte... on doit se tromper et oui, des fois on se trompe et de mesurer assez vite, et assez précisément... quel est l'impact de la pathologie, de la maladie, de la souffrance la douleur, dans le quotidien. Je crois qu'il faut vite dépasser l'aspect évaluation quantitative, je vais l'ouvrir à trois, je vais l'ouvrir à cinq, ça peut apporter un plus, mais, je crois que l'important c'est de se rendre compte quelle est la conséquence dans la vie quotidienne.

P2 : Oui il y a une interaction quand même. On ne peut pas expliquer en détail en se concentrant sur ces techniques. Mais si on se concentre uniquement sur ces techniques, on passe à l'as le relationnel et c'est pas bon non plus. C'est très bizarre, ça. C'est des compromis tout le temps. C'est pour ça qu'il faut quand même du temps, car le temps de traitement n'est pas si important que ç... Le temps de toucher n'est pas énorme. Le traitement il dure un certain temps, mais le toucher, il y en a beaucoup moins.

Le remplaçant manifeste ici son dépit, face à l'incompréhension par le patient de la logique thérapeutique sous-jacente à la posture qu'il lui fait adopter. II lui a pourtant "expliqué plusieurs fois ». Le praticien souligne au contraire la nécessité de faire quelque chose de cette incompréhension. Pour lui en effet, l'action, toujours à élucider pour le patient, se développe dans l'échange avec ce dernier. Ce qui le conduit à dépasser la résistance du patient face à une mobilisation douloureuse, mais dont l'effet thérapeutique est avéré. 
R2 : Là, je ne lui rappelle pas la consigne, il monte le bras mais après, il faut penser qu'en montant le bras, il faut descendre l'épaule, c'est pas ce qu'il faut faire ; je lui ai déjà expliqué plusieurs fois...parce qu'il y avait une histoire de proprioception et que, comme il avait été immobilisé, il avait une épaule qui partait...

P2 : Oui, mais moi... C'est un exercice que je fais faire à chaque fois pour les épaules parce que les patients ne le font jamais totalement bien et je ne l'ai pas encore fait avec lui. Le temps passait, donc je vais voir dans cette position dite nec plus ultra pour l'épaule si on a cette espèce de tendon qui glisse; en fait c'est mon préféré par rapport au patient qui le redoute et qui disent "non! pas celui-là! » et moi qui dit « et si, moi je l'aime bien!»

Ici se contredisent les deux types de modèles: celui, cognitif, du relationnel à assurer (« il y a une interaction quand même ; je lui explique... »), parfois contradictoire avec celui, opératif, des nécessités du traitement ("c'est mon préféré par rapport au patient qui le redoute »). La convergence implicitement recherchée entre perception, conceptualisation et action, instrumentée dans la relation thérapeutique, est ici prise en défaut.

Ainsi les modèles cognitif et opératif convergent, s'ignorent ou entrent en contradiction ; cet espace de jeu entre les modèles manipulés par les professionnels est aussi un espace discrétionnaire d'apprentissages. La circulation entre ces deux modèles cognitif et opératif, est fonction d'un "format " - au sens de J. S. Bruner (1983, p. 22) - qui configure l'interaction communicative selon la participation associée aux exigences de la situation (prescription, tâches et leur contexte) et l'engagement des ressources du sujetremplaçant.

\section{Cadre d'exercice et position de remplaçant : les marques de l'engagement}

Les éléments recueillis, relatifs à une pré-enquête quantitative exploitant les communications avec le patient en séance (Olry et al., 2005), ont souligné les formes de communication les plus usitées par les soignants qui consistent en :

- des échanges asymétriques d'informations; en effet, «le » patient est majoritairement dans une posture de délégation de son déficit au praticien et pose peu des questions, tandis qu'il produit des réponses riches en informations;

- des prises d'informations sous forme de questions, plus ou moins nombreuses selon le profil du patient (la nature du déficit, le type de traitement, ses dispositions au soin, l'enrôlement obtenu, etc.) ;

- des validations des propos des patients sous une forme empathique. Dès lors, si les encouragements, peu nombreux, sont une voie d'engagement du patient dans le soin, les critiques sont majoritairement absentes du discours du praticien pourtant soucieux de l'éduquer ;

- la diffusion de consignes pour la réalisation des prescriptions et l'engagement du patient dans le soin.

Chaque soignant s'ajuste ainsi au cas du patient et oriente la définition du «problème » et le diagnostic qui en découle. II rapporte ainsi chaque cas particulier à une classe de pratiques de soin, tout en envisageant les développements de la thérapeutique au regard de la durée allouée par la prescription.

Le praticien ne les tient pas pour négligeables, dans la mesure où elles le renseignent sur les attentes du patient. II les prend même d'autant plus au sérieux que l'engagement du patient dans le traitement est conditionné par le dépassement de ce déficit perçu.

Toutefois, le contenu des communications ne laisse pas de surprendre puisque les prises de parole relevant d'interactions sociales, de civilité, sont majoritaires. Ainsi les interactions visant à expliquer l'origine des symptômes et des douleurs du patient sont minoritaires, sauf à relever de la légitimité d'un paramédical : le climat, les conditions de travail sont les plus fréquemment évoquées. Les caractéristiques de la douleur (localisation, plus ou moins aiguë/sourde, moment d'apparition, etc.) sont certes brièvement évoquées, mais tout ce passe comme si c'était dans les propos généraux que s'énonçaient, se repéraient les indices particuliers utiles à la conduite du soin. En effet, les connaissances scientifiques concernant le fonctionnement dégradé du corps humain ne sont que rarement exposées.

Enfin, si les résultats de la globalité des séances et l'ordonnancement de chacune d'elle font l'objet d'interactions avec le patient, les buts des séances en sont très rarement l'objet. Lorsque le praticien s'exprime, c'est pour dire ce que le patient doit faire dans l'instant. De même, peu d'entre eux s'attachent à préparer le travail de rééducation hors séance.

Les remplaçants sont dans une tout autre posture. Chaque patient est un défi posé à leur sagacité. Il s'agit pour eux de dépasser la «part d'ombre » (Nyssen \& De Keyser, 1993, p. 244) constituée par la variabilité des contraintes et des conditions de réalisation 
de la tâche portées par le patient. C'est en ce sens qu'on peut parler d'une performance, comme atteinte d'un niveau pratique où les limites du corps et du savoir-faire sont mises à l'épreuve dans un cadre de déstabilisation cognitive ou expérientielle. S'y ajoute l'idée de montrer qu'on réussit à faire face à cet inédit.

En termes de dispositif, les remplaçants construisent l'échange qui leur paraît d'abord adapté à euxmêmes. II se fonde sur une investigation personnelle :

- qui vise à entendre les difficultés pratiques des patients, formulées en attente de résultat «je n'arrive pas à tenir mon téléphone à l'oreille », «je monte difficilement les trois marches du perron »;

- d'un briefing attendu du patient sur la gêne ressentie et ses capacités restantes, censé renforcer l'efficience de la démarche par la modulation des exigences de soin sur le plan thérapeutique ;

- sur la base de la prescription, le soignant se livre dès l'accueil, à une chasse aux indices orientée tout à la fois vers le corps du patient, son discours et vers ses propres connaissances.

Cette enquête s'appuie sur leur expérience individuelle combinant des ressources formelles (mesures) et informelles, qui mobilisent l'analyse rétrospective d'actions antérieures.

En terme de contenus, l'activité du remplaçant présente donc des caractéristiques spécifiques, et notamment :

- le processus de contrôle de ses propres actions est plus poussé et donc le volume de questions à son initiative est supérieur à celui du praticien ;

- l'incertitude relative sur le cas/patient, l'amène à investir plutôt une modalité de soin susceptible d'appréhender par une procédure stable une variété de situations de soin (14), puisqu'il « ne voit pas la fin » du traitement ;

- cette application de protocoles «impersonnels» stabilisés, formalisés, instrumentés est plus orientée par la satisfaction immédiate du client que vers l'exécution appliquée de la prescription.

Les échanges des soignants avec les patients en séance jouent différentes fonctions : ponctuer l'action, orienter le patient sur une sensation, une posture, une focalisation précise en lien à un corps symboliquement structuré. Ce faisant, elles construisent de la situation, laquelle n'est pas construite de la même façon selon la position tenue :
$\mathrm{R} 1$ : « je ne suis pas d'avis que la parole participe au soin » (Auto-confrontation)

puis avec le praticien

R1: " le fait d'écouter le patient, de parler avec lui permet de faire une enquête pour savoir d'où vient le problème, éviter qu'il se reproduise, etc. " (Autoconfrontation croisée).

Ce remplaçant construit en deux temps son rapport à l'usage de la parole du patient dans le soin : ce qui lui apparaît d'abord à la périphérie de l'action, devient à la réflexion un moyen de conduite de la thérapeutique. Au travers des débats entendus, les formalisations énoncées fondent une mémoire du corps (Faure, 2002) sur l'appropriation partagée d'un geste technique, qui de plus établit ainsi un rapport de reconnaissance entre soignants.

Notre interprétation est ici que le contenu des échanges verbaux ne participe pas toujours de l'efficacité thérapeutique, mais contribue à construire de la situation, c'est-à-dire un cadre à la coordination des modèles opératif et cognitif.

\section{LA FORMALISATION : PRODUIT DE L'ACTIVITÉ, EFFET D'APPRENTISSAGE}

Dans le cadre si spécifique des apprentissages au travail, leur dimension informelle ne peut paradoxalement être affirmée qu'au regard de leur «formalisation " accidentelle ou provoquée. Dans le cas des situations de remplacement en kinésithérapie, et dans des conditions de participation qui le permettent, notre étude montre que les formalisations émergentes révèlent des objets d'apprentissage relevant plutôt de connaissances méta-fonctionnelles (Falzon, 1994), qui construisent les conditions de l'activité fonctionnelle. Les processus qui les fondent ne sont pas sans logiques que nous proposons maintenant de dégager.

\section{Logique du cadre : construire le milieu}

Le premier processus qui concourt à une mise en forme d'apprentissages antérieurs est la confrontation du monde de référence des remplaçants et des praticiens. En effet, pour le choix d'un protocole, pour l'adoption d'une technique, les remplaçants « hésitent » entre deux options : suivre et réagir aux événements d'une part et d'autre part, "vivre selon 
l'idée » (Badiou, 2006), i. e. agir en conformité avec la compréhension qu'ils se font de la situation du patient.

Dans la première perspective, le symptôme du patient se signale comme événement clinique, rupture signifiante au regard du système de cohésion des conséquences qui le rend possible. Dès lors, le patient est réduit à l'événement clinique, une dysfonction, qui permet au praticien de mettre en œuvre le traitement selon le protocole prévu.

Dans la seconde perspective, l'événement clinique est une occasion d'acquérir une capacité de décision par des anticipations subjectives au regard d'un corps de conséquences plus ou moins connues. L'événement ne vaut pas pour lui-même mais en ce qu'il crée de nouvelles conditions pour faire naître une décision.

Tel que nous venons de le présenter, il s'agit bien d'un processus qui en disposant sujet et événement à chaque séance "autrement ", contribue à la formalisation d'apprentissages constitutifs d'un monde, cadre qui permet de voir (et parfois de ne pas voir). Si elle structure les relations de dépendance et relation d'ordre entre des facteurs, cette manière d'opérer et de se constituer des points de vue sur le monde est dépendante des conditions de participation du remplaçant.

\section{Logique de participation : coordonner des buts et des motifs}

Le cadre de référence de l'action n'est pas exclusivement orienté par l'objet de l'action thérapeutique mais tout autant, sinon davantage, par la situation sociale peu familière au remplaçant. Celui-ci est en effet confronté à un environnement dont il doit s'accommoder. Chaque situation, les soignants ne manquent pas de le souligner, est spécifique. Mais cette singularité réside dans l'agencement, la composition d'éléments, de variables qui, eux, se retrouvent dans d'autres situations, parce que déterminés par des fonctionnements sociaux, économiques qui ne dépendent pas des situations, même s'ils s'y actualisent.

Chaque situation apparaît donc comme un composé unique de variables qui ne le sont pas. Mais chaque situation est aussi le fait de sujets qui s'y engagent différemment selon le sens qu'ils lui donnent et les ressources dont ils disposent face aux exigences de la tâche. Le dispositif de participation du remplaçant croise ainsi plusieurs niveaux de la situation :
- celui du cas/patient, objet du recueil d'information, de la production de significations, de l'action éventuelle ;

- celui du remplacement institutionnellement cadré, subjectivement redéfini à travers le «format de coordination" que chaque soignant lui donne, notamment au regard des règles de communication qui le situe (Cicourel, 2002) ;

- celui du patient enfin, destinataire de l'activité du soignant.

Chacun de ces niveaux de la situation appelle des critères de pertinence et de recevabilité des significations accordées à cette représentation de la réalité.

Pour ce faire, les soignants mobilisent des ressources non formelles telles que les profils de patients et de praticiens, rarement évoqués, mais sollicitant des modèles d'action appropriés. On voit ici comment l'activité du remplaçant s'organise d'une part en élaborant des significations, d'autre part en mobilisant des ressources.

La coordination significations/ressources construit la situation, conçue comme configuration (15) dans les dynamiques subjectives et sociales. La dynamique du but est liée aux "formes régulières » et aux types de singularités qui construisent la situation. Par ailleurs elle s'inscrit également dans la confrontation du monde (Béguin, 2004) du remplaçant, et des formats, constitués des interactions et instituant de l'échange (Bruner, 1983) avec le praticien, ce que mettent en évidence les motifs invoqués.

Ce faisant, la mobilisation de significations semble orientée dans l'activité vers l'identification des situations. C'est moins donc une expertise particulière dans l'analyse du cas patient défini comme l'objet de l'action, que la construction d'une représentation des aspects de cette réalité qui satisfait des critères de pertinence dans la situation sociale du remplacement.

\section{Logique de l'engagement : concilier l'activité et son mode d'appréciation}

La participation n'apparaît plus seulement alors comme un dispositif social mais comme un mode d'appréciation, au regard d'un format (corporel, verbal) de ce qui fait information.

C'est d'abord la conscience de la situation, au sens des paramètres pertinents pour percevoir, agir et anticiper l'action avec un patient plus ou moins 
enrôlé, donc plus ou moins engagé dans une thérapeutique dont le corps est à la fois l'objet et l'unité agissante. À cet égard, la clarté des exigences de sa tâche constitue pour le remplaçant l'enveloppe (difficultés et opportunités) dans laquelle se déploie son activité, elle-même fonction de ses capacités d'action.

C'est ensuite la capacité thérapeutique des soignants à passer de l'incapacité perçue, entrave aux mouvements quotidiens, à la déficience, état clinique dont l'analyse est le fruit d'une investigation commune avec le patient. Cette capacité à faire le lien entre un perçu et un état clinique structure les finalités de la thérapeutique retenue. La formalisation est d'abord ici une théorisation pragmatique qui donne sa valeur au dispositif thérapeutique, ne séparant pas le déficit de son origine, base même de toute nouvelle constitution. Une fois formalisée, la déficience renforce ses liens avec ses bases informelles pour donner naissance à une structure actualisée de la situation.

C'est enfin l'acte thérapeutique assisté du patient, qui caractérise un format croisant enrôlement physique du patient et engagement des savoirs experts du soignant.

C'est dans l'articulation format/activité qu'émergent des apprentissages informels, par un double étayage - dimensions subjective et sociale du travail - que la notion de situation permet d'appréhender.

En d'autres termes, l'activité d'interprétation que représente chaque cas est une "épreuve de réalité " (Thévenot, 2006) qui suppose des capacités conjointement attendues du remplaçant et du milieu dans lequel il s'engage.

\section{CONCLUSION}

Dans cette étude ont d'abord été présenté trois contextes du travail kinésithérapique qui inscrivent la relation de soin dans un dispositif de participation du remplaçant.

Nous avons ensuite présenté quelques données empiriques comparatives, dont les différences dans la hiérarchisation des buts énoncés, soulignent un engagement dépendant de deux cadres d'un même monde : celui du mouvement d'un corps à restaurer dans un monde déterminé ; celui de l'idée d'un patient à re-éduquer au regard de la déficience que lui-même perçoit. L'action thérapeutique illustre ainsi l'idée que la formalisation ne relève pas seulement d'un modèle cognitif mais également d'un modèle opératif, social, qui expliquent pour une part la réussite du traitement. Le passage de l'un à l'autre de ces modèles largement non-formalisés est producteur d'apprentissage.

La formalisation est travaillée dans l'interaction entre praticien et remplaçant ; elle n'obéit pas à une logique unique, mais à un faisceau de processus dont la conjonction est le but du traitement. Les représentations pour l'action, les schémas et scénarios, les conventions établies, instruments du praticien sont des ressources pour le remplaçant dès lors qu'elles prennent sens au regard des modèles mobilisés. Ce qu'un soignant a marqué d'un glissement de terme, présentant son métier moins comme de la rééducation que comme de la réhabilitation (rétablir quelqu'un dans son premier état de sujet).

Paul Olry paul.olry@cnam.fr Conservatoire national des arts et métiers

\section{NOTES}

(1) L'apprentissage informel serait un " apprentissage découlant des activités de la vie quotidienne liées au travail, à la famille ou aux loisirs. II n'est pas structuré (en termes d'objectifs, de temps ou de ressources) et n'est généralement pas validé par un titre. L'apprentissage informel peut avoir un caractère intentionnel, mais, dans la plupart des cas, il est non-intentionnel (ou fortuit/ aléatoire) 》 (Brougère \& Bézille, 2007, p. 138). "Pour n'être pas réalisé dans un espace spécifique, l'apprentissage non-formel est cependant structuré (en termes d'objectifs, de temps ou de ressources). L'apprentissage non-formel est intentionnel de la part de l'apprenant. » (Ibid., p. 128)

(2) Les 45000 kinésithérapeutes se caractérisent par un exercice à $80 \%$ libéral et par une fréquentation de la formation continue estimée à moins de $50 \%$ (Chiffres : ministère de la Santé, Direction de la recherche, des études, de l'évaluation et des statistiques, 2003).

(3) Par commodité, et pour distinguer nos désignations, le professionnel titulaire sera désigné comme «le praticien »; si nous par- lons du remplaçant et du praticien ensemble, nous dirons « soignants".

(4) Définie comme «force, puissance, vertu qui produit l'effet qu'on en attend "selon le dictionnaire de l'Académie.

(5) Ce dont les logiques curriculaires des instituts de formation (K1, $\mathrm{K} 2, \mathrm{~K} 3$ ) témoignent.

(6) Ainsi, la « distance au patient », sans cesse à trouver pour le praticien, constitue un axe peu travaillé en formation initiale, tant il est vrai qu'elle fait partie de ces compétences incorporées qui s'acquièrent par l'expérience et qui sont difficilement transmissibles.

(7) Dans le cas des masseurs-kinésithérapeutes et pour ne prendre que les travaux sur les lombalgies par exemple, les professionnels reconnaissent que de nombreux éléments leur manque pour comprendre l'efficacité des traitements qu'ils appliquent. En France, la conférence de consensus sur ce thème souligne cette carence d'études cliniques (Fournier et al., 2007). 
(8) On consultera avec intérêt à cet égard le site internet de l'Association francaise pour la recherche et l'évaluation en kinésithérapie [http://www.afrek.org/, consulté le 8 novembre 2007] qui propose des fiches de bilan, de synthèse de diagnostic et des outils d'évaluation.

(9) Ainsi, il arrive plus rarement que le patient se mêle au débat, la chronicité vécue du déficit faisant de lui un " expert " de sa rééducation. II manifeste une plus ou moins grande connaissance pragmatique des éléments de savoirs constitutifs du soin pertinent, et son attente d'une prise en charge par le soignant sonne comme une réponse à l'enrôlement que ce dernier tente d'obtenir de lui.

(10) Le matériel empirique de notre étude est ainsi composé d'observations filmées, objet d'auto-confrontations croisées entre un praticien et son remplaçant (Clot \& Faïta, 2000). Les séances observées ont concerné des rééducations de l'épaule par trois remplaçant(e)s et leur commentaire, croisé avec celui des praticiens.
(11) « Les situations sont ce à quoi les sujets s'ajustent via les définitions qu'ils en donnent ; ce faisant leur réalité vient de ces définitions " (Ogien \& Quéré, 2005)

(12) Nous entendons ici "information " au sens actif d' "enquête que l'on mène pour constater un fait, pour s'assurer de la vérité d'une chose ».

(13) Dans les exemples qui suivent on trouvera les initiales $P$ pour praticien et $R$ pour remplaçant, les chiffres indexant les dyades P/R.

(14) Cet intitulé large veut souligner l'importance du versant social de l'enjeu de prise en charge par rapport à son versant thérapeutique.

(15) La notion de configuration est utilisée, là, dans l'usage qu'en a précisé J.-M. Barbier (1999) comme « mobilisation singulière de formes régulières ».

\section{BIBLIOGRAPHIE}

Badiou A. (2006). Logique des mondes. Paris : Éd. du Seuil.

BARBieR J.-M. \& GalATANU O. (1999). " La singularité des actions: quelques outils d'analyse ". In J.-M. Barbier (éd.), L'analyse de la singularité de l'action. Paris : PUF, p. 13-51.

Beguin P. (2004). "Mondes, monde commun et versions des mondes ", Bulletin de psychologie, vol. $57, \mathrm{n}^{\circ} 1$ (n $\left.{ }^{\circ} 469\right)$, p. $45-48$.

BILLETT S. (2001) Critiquing workplace learning discourses: participation and continuity at work. Document disponible au format HTML sur Internet à l'adresse: http:// www.infed.org/archives/e-texts/billett_workplace_ learning.htm (consulté le 8 novembre 2007).

BoltANSKI L. \& ThÉVEnOt L. (1991). De la justification: les économies de la grandeur. Paris : Gallimard.

Boreham N. ; Samurçay R. \& Fischer M. [éd.] (2002). Work Process Knowledge. London: Taylor \& Francis.

Brougère G. \& BézILLE H. (2007). « De l'usage de la notion d'informel dans le champ de l'éducation ". Revue française de pédagogie : recherches en éducation, $\mathrm{n}^{\circ} 158$, p. 117-160.

BRUNeR J. S. (1983). Le développement de l'enfant : savoir faire, savoir dire. Paris : PUF.

Carré P. \& Charbonnier O. (2003). Les apprentissages professionnels informels. Paris : L'Harmattan.

Cicourel A. (2002). Le raisonnement médical, une approche sociocognitive. Paris : Éd. du Seuil.

CLot Y. \& FAïTA D. (2000). "Genres et styles en analyse du travail : concepts et Méthodes ». Travailler, $\mathrm{n}^{\circ}$ 4, p. 7-42.

FALzoN P. (1994). "Les activités méta-fonctionnelles et leur assistance ". Le travail humain, t. $57, \mathrm{n}^{\circ} 1, \mathrm{p} .1-23$.

FAURE S. (2000). Apprendre par corps: socio-anthropologie de la danse. Paris : Éd. La Dispute.

Fournier C.; Jullien-Narboux S.; Pélicand J. \& VinCEnT I. (2007). "Modèles sous-jacents à l'éducation des patients ". Évolutions : résultats d'études et de recherches en prévention et en éducation pour la santé [Institut national de prévention et d'éducation pour la santé], $\mathrm{n}^{\circ} 5$, janvier, $8 \mathrm{p}$.

Gadrey J. \& De BANDT (1994). La relation de service. Paris : Presses du CNRS.
Hughes E. C. (2003). "La fabrication d'un médecin ». Les sciences de l'éducation pour l'Ėre nouvelle, vol. 36, $\mathrm{n}^{\circ} 2$, p. $57-70$.

Houel A. (2002). "Des femmes face à un métier masculin : représentations et résistances ". Éducation permanente, $\mathrm{n}^{\circ} 150$, p. 43-51.

NYSSEN A.-S. \& De KeYser V. (1993). "L'erreur humaine en anesthésie ». Le travail humain, vol. 56, n 2-3, p. 243-266.

OGIEN A. \& QUÉRÉ L. (2005). Le vocabulaire de la sociologie de l'action. Paris : Ellipses.

OLRY P. (2003). « Organisation du soin et développement des compétences: contribution à la professionnalisation en masso-kinésithérapie ". Les sciences de l'éducation pour l'Ére nouvelle, vol. 36, $\mathrm{n}^{\circ}$ 2, p. 91-106.

OLRY P. (2004). «Un dispositif de recherche sur les pratiques de métier: le cas des masseurs-kinésithérapeutes». In J.-M. Barbier (éd.), Les savoirs d'action, une mise en mots des compétences. Paris: L'Harmattan, p. $169-184$.

OlRY P. \& BAUTZER E. (2003). « Diagnostic kinésithérapique. Les conditions d'une coproduction ". Recherche et formation pour les professionnels de l'éducation, $\mathrm{n}^{\circ} 42$, p. $35-50$

OlRY P. et al. (2005). "Distance thérapeutique et corps à corps ", Éducation permanente, n 165 , p. 42-54.

PAstRÉ P. \& Weill-FAssina A. (2004). « Les compétences professionnelles et leur développement ». In P. Falzon (dir.), Ergonomie. Paris : PUF, p. 213-231.

Pastré P. ; Mayen P. \& Vergnaud G. (2006). "La didactique professionnelle ". Revue française de pédagogie recherches en éducation, $\mathrm{n}^{\circ} 154$, p. 145-198.

SONNTAG M. (2002). "Les schémas d'action, outil de figuration des représentations dans l'analyse des pratiques professionnelles ». Revue française de pédagogie, $\mathrm{n}^{\circ} 138$, p. 29-39.

THÉVENOT L. (1999). «L'action comme engagement ». In J.-M. Barbier (éd.), L'analyse de la singularité de l'action. Paris : PUF, p. 213-238.

THÉVENOT L. (2006). L'action au pluriel : sociologie des régimes d'engagement. Paris : Éd. La Découverte. 